\title{
Promising Neuroimaging Biomarkers in Depression
}

\author{
Chien-Han Lai ${ }^{1,2,3} \bowtie$ \\ ${ }^{1}$ Institute of Biophotonics, National Yang-Ming University, Taipei, Taiwan \\ ${ }^{2}$ Psychiatry \& Neuroscience Clinic, Taoyuan, Taiwan \\ ${ }^{3}$ Department of Psychiatry, Yeezen General Hospital, Taoyuan, Taiwan
}

\begin{abstract}
The neuroimaging has been applied in the study of pathophysiology in major depressive disorder (MDD). In this review article, several kinds of methodologies of neuroimaging would be discussed to summarize the promising biomarkers in MDD. For the magnetic resonance imaging (MRI) and magnetoencephalography field, the literature review showed the potentially promising roles of frontal lobes, such as anterior cingulate cortex (ACC), dorsolateral prefrontal cortex (DLPFC) and orbitofrontal cortex (OFC). In addition, the limbic regions, such as hippocampus and amygdala, might be the potentially promising biomarkers for MDD. The structures and functions of ACC, DLPFC, OFC, amygdala and hippocampus might be confirmed as the biomarkers for the prediction of antidepressant treatment responses and for the pathophysiology of MDD. The functions of cognitive control and emotion regulation of these regions might be crucial for the establishment of biomarkers. The near-infrared spectroscopy studies demonstrated that blood flow in the frontal lobe, such as the DLPFC and OFC, might be the biomarkers for the field of near-infrared spectroscopy. The electroencephalography also supported the promising role of frontal regions, such as the ACC, DLPFC and OFC in the biomarker exploration, especially for the sleep electroencephalogram to detect biomarkers in MDD. The positron emission tomography (PET) and single-photon emission computed tomography (SPECT) in MDD demonstrated the promising biomarkers for the frontal and limbic regions, such as ACC, DLPFC and amygdala. However, additional findings in brainstem and midbrain were also found in PET and SPECT. The promising neuroimaging biomarkers of MDD seemed focused in the fronto-limbic regions.

Psychiatry Investig 2019;16(9):662-670
\end{abstract}

Key Words Major depressive disorder, Anterior cingulate cortex, Dorsolateral prefrontal cortex, Orbitofrontal cortex, Hippocampus, Fronto-limbic.

\section{INTRODUCTION}

Major depressive disorder (MDD) is an important psychiatric illness with substantial impacts in many perspectives of function and life quality. The functional impairments were correlated with the clinical symptoms. ${ }^{1}$ The physical, psychological and social domains of life quality is inter-related to functional status. ${ }^{2}$ MDD symptoms would interfere with concentration, motivation and cognitive functions, which would cause patients to have daily functional impairments. ${ }^{3}$ Therefore, it is important for scientists and clinicians to understand the pathophysiology of MDD. Many articles about the structural and functional pathophysiology of MDD mentioned the

Received: June 12, 2019 Accepted: July 25, 2019

$\triangle$ Correspondence: Chien-Han Lai, MD, PhD

Institute of Biophotonics, National Yang-Ming University, No.155, Sec.2, Linong Street, Taipei, 112 Taiwan (ROC)

Tel: +886-2-2826-7000, Fax: +886-2-2823-5460

E-mail: stephenlai99@gmail.com

(c) This is an Open Access article distributed under the terms of the Creative Commons Attribution Non-Commercial License (https://creativecommons.org/licenses/bync/4.0) which permits unrestricted non-commercial use, distribution, and reproduction in any medium, provided the original work is properly cited. possible roles of fronto-limbic network. ${ }^{4-7}$ The fronto-limbic network seemed to originated from the initial "limbic-corticalstriatal-pallidal-thalamic tract" model for MDD. ${ }^{8}$ Recently an evolved MDD model mentioned the importance of ventromedial prefrontal cortex, anterior cingulate cortex (ACC) and lateral parietal cortex, which might be associated with self-referential problems and negative ruminations. ${ }^{9}$

In the pathophysiology model, ACC seemed to play a significant role in MDD. ACC was also responsible for important functions of $\mathrm{MDD}$, such as the attention, problem solving, motivation, decision making. ${ }^{10-12}$ Two components of ACC, affective and cognitive subdivisions, ${ }^{11,13}$ might be associated with functional impairments in MDD. The affective subdivision was connected with limbic regions, such as the amygdala and brainstem, to modulate the emotions. ${ }^{14}$ The cognitive subdivision was involved in cognitive processing of MDD. ${ }^{13}$ The cognitive and affective components of ACC might have important roles in the pathophysiology of MDD. ${ }^{15,16}$ Therefore the MDD pathophysiology biomarkers might include the ACC. In addition to the ACC, other regions of frontal lobe, such as dorsolateral prefrontal cortex (DLPFC), ${ }^{17-20}$ would be 
crucial for the cognitive control of depression symptoms. The emotional responses and depression symptoms might be originated from the limbic regions, such as the hippocampus and amygdala. ${ }^{8,18,21-25}$ From the viewpoint of cognitive control of emotional responses, the fronto-limbic network or circuit would be the state-of-art model to explain the influences of cognition impairments and the exaggerated negative emotion responses from the limbic regions in MDD.

However, the promising neuroimaging biomarkers in MDD were still not conclusive according to the extent and focus of current study. In this article, I would focus on the literature review of neuroimaging in the study of pathophysiology of MDD, including the magnetic resonance imaging (MRI), magnetoencephalography (MEG), near-infrared spectroscopy (NIRS), electroencephalography (EEG), positron emission tomography (PET), single-photon emission computed tomography (SPECT). The potentially promising biomarkers for the neuroimaging perspectives would be discussed and summarized to establish a possibly conclusive biomarker model for MDD.

\section{MDD biomarkers revealed by MRI and MRS}

The MRI part of neuroimaging biomarkers would include the magnetic resonance spectroscopy (MRS), structural and functional MRI. The MRS can measure the neural metabolites inside the brain, such as the $\mathrm{N}$-acetyl aspartate and choline. The MRS study of adolescent melancholic MDD depression patients had significant correlations between tryptophan metabolites and neurotoxic metabolites in the limbic regions, such as the striatal areas. ${ }^{26} \mathrm{~A}$ study using functional MRI integrated classifiers showed better predictability and accuracy for the detection of biomarker areas involving the emotion processing area. ${ }^{27}$ The visual processing of emotional faces in MDD showed limbic activations, especially in the hippocampus. ${ }^{28}$ The pre-school children MDD also had heightened responses to sad faces in the limbic regions, such as the amygdala. ${ }^{29}$ The disrupted amygdala activity during face emotion identification in depressed children around 4-6 years old, which suggested the potential biomarker role of amygdala in endogenous depression. ${ }^{30}$ The genome-wide neuroimaging study showed a link between a potential candidate of specific genotype and hippocampus volume, which suggested the complex relationship between genotype and MRI biomarkers in a more comprehensive model for biomarkers in MDD. ${ }^{31}$ The baseline brain activities would be fluctuated in the hippocampus of MDD patients with suicide ideation. ${ }^{32}$ The hippocampus was also the state-dependent biomarker region for antidepressant treatment in MDD. ${ }^{33}$ The recurrent MDD patients also had lower gray matter volume (GMV) in the left hippocampus. ${ }^{34}$ The hippocampus-related neural network, including the DLPFC, was also altered in the young subjects with family history of MDD, especially during a memory task. ${ }^{35}$ The hippocampus tail volumes were relatively larger in MDD and the hippocampus tail volumes would be associated with clinical remission after antidepressant treatment. ${ }^{36}$

The frontal dysfunction has also been replicated in many MRI studies of MDD. A MRS study showed an increased choline/creatine ratio in the ACC of MDD patients, which might be a possible diagnostic aid tool. ${ }^{37} \mathrm{~A}$ review article of MRS study in MDD showed higher choline levels in the frontal lobe of depression patients. ${ }^{38} \mathrm{MDD}$ could also be differentiated from the panic disorder using regional homogeneity pattern in the left ACC. ${ }^{39}$ The meta-analysis of GMV studies in MDD also revealed the GMV alterations in bilateral ACC. ${ }^{40} \mathrm{~A}$ meta-analysis of resting-state functional MRI studies suggested a possible pattern of diagnosis classification according to the hyperactivity/hyper-connectivity between ACC and other prefrontal regions for the external-directed cognition. ${ }^{41}$ The alterations in cortisol awakening responses, which would influence the hypothalamic-pituitary-adrenal axis homeostasis in MDD, were also associated with reduced GMV and increased stress-related brain activities in the ACC, ${ }^{42}$ which also corresponded to another GMV study in MDD with panic disorder. ${ }^{6}$ A machine-learning algorithm study showed the crucial roles of areas for the guilt-selective neural signature, such as the ACC, hippocampus and thalamus. ${ }^{43}$ The regional homogeneity of ACC might be associated with illness duration and depression severity in MDD patients. ${ }^{44}$ The higher fluctuations of resting-state brain activity in the subgenual ACC of MDD patients would be significantly reduced after electroconvulsive therapy. In addition, the subgenual ACC activity would predict the treatment responses with the reductions in the connectivity between ACC, hippocampus and other prefrontal regions. ${ }^{45}$ In addition, the variants of ACC thickness would predict the responses to electroconvulsive therapy ${ }^{46}$ which corresponded to that dorsal part of ACC (cognitive subdivision of ACC) volume could predict the long-term clinical outcome trajectory ${ }^{47}$ and prediction of rostral ACC thickness for the treatment response to transcranial magnetic stimulation in $\mathrm{MDD} .^{48}$ The micro-integrity of cingulate bundle also predicted the treatment responses of MDD patients for antidepressants. ${ }^{49}$ The blunted response of ACC during consummation phase and that of hippocampus during effort phase of functional MRI reward task also predicted the high risk for adolescent MDD..$^{50} \mathrm{~A}$ review article suggested that the pharmaceutical interventions would relieve functional connectivity alterations in the fronto-limbic, default mode network and parieto-temporal regions. The psychotherapeutic interventions have also been showed to influence the functional connectivity within 
the fronto-limbic network. The electroconvulsive and magnetic stimulation therapy would also alter the ACC, DLPFC and default mode network. ${ }^{51}$ The depression severity was associated with elevated connectivity between fronto-limbic regions, such as ACC, amygdala and hippocampus, in a resting-state functional MRI study. ${ }^{52}$ The distinct differences of functional connectivity in the fronto-limbic network between suicide ideators and suicide attempters were also found in the resting-state functional MRI study. ${ }^{53} \mathrm{~A}$ meta-analysis of machine learning studies in MDD also confirmed the importance of ACC for the treatment prediction and the crucial role of fronto-limbic network for the pathophysiology of MDD. ${ }^{54}$ A review of structural MRI studies in MDD also supported the important roles of fronto-limbic network for the pathophysiology. ${ }^{55}$ The white matter tract study in MDD also showed the alterations in the micro-integrity of superior longitudinal fasciculus, which connected the frontal regions with the limbic regions and temporal regions. ${ }^{5,57}$ It also supported the fronto-limbic model for the biomarker regions in MDD. The core region of the fronto-limbic network might be the ACC. ${ }^{58}$

The magnetic seizure therapy-related cortical inhibitions of frontal cortex were the significant indicators of remission for suicidal ideation. ${ }^{59}$ The prefrontal circuit dysfunction, such as the hypo-activations of DLPFC during the working memory updating and conscious negative emotion processing tasks of functional MRI experiments.$^{60}$ The prefrontal cortex-related cognitive control and emotion regulation abilities would be associated with resilience factors in the treatment response. ${ }^{61}$ The persistent alterations of anterior subnetwork of default mode network, which also included frontal regions, were observed in the MDD patients after antidepressant treatment. ${ }^{62}$ The implantation of deep brain stimulation target and the transcranial repetitive magnetic stimulation in the left DLPFC would relieve the depression symptoms in the treatment-resistant MDD adolescents. ${ }^{63}$ The DLPFC was also a popular treatment target of brain stimulation in MDD. ${ }^{64}$ The DLPFC and dorsal ACC cognitive subdivision could form a cognitive control network. The attenuation of intrinsic activity in cognitive control network would predict the treatment response in remitted MDD patients. ${ }^{65}$ The transcranial magnetic stimulation in the DLPFC would also trigger higher activities of subgenual ACC in the treatment of MDD patients, which would modulate the cognitive control network. ${ }^{66}$ The functional MRI-related global brain signal regression was more significant in the DLPFC in treatment-resistant MDD patients, which could be normalized by ketamine infusion treatment. ${ }^{67}$ The non-remitted late-life MDD patients had significantly greater reductions in the GMV of orbitofrontal cortex (OFC) than remitted latelife MDD patients and controls. ${ }^{68}$ The GMV of OFC also predicted the antidepressant treatment responses in MDD. ${ }^{34}$ The deficient inhibition of return for emotional materials was also altered in the OFC and hippocampus-related activity and functional connectivity of MDD patients, which suggested that OFC might be specific for remitted responses and hippocampus might be specific for depressive phase. ${ }^{69}$

However, some studies were still against the significant roles of fronto-limbic regions as biomarkers of MDD. A MRS study showed that $\mathrm{N}$-acetyl aspartate levels showed no significant changes in the DLPFC and amygdala of first-episode MDD patients after antidepressant treatment. ${ }^{70} \mathrm{~A}$ meta-analysis showed a lack of increases in the brain-derived neurotrophic factor after non-invasive brain stimulation in the DLPFC of MDD patients. ${ }^{71}$ In addition, the hippocampus enlargement might not represent a sole predictor for the treatment response to electroconvulsive therapy. ${ }^{72}$

In summary, for the MRI and MRS study in MDD, the most promising biomarkers might be the ACC. The other prefrontal regions, such as DLPFC and OFC, may cooperate with ACC to contribute to the inhibition of emotional response and emotional memory via the cognitive control network. The emotional response and memory might be originated from the limbic regions, such as the amygdala and hippocampus. The model of neuroimaging biomarkers in MRI and MRS may still exist in the architecture of fronto-limbic model. In addition, the prediction of treatment response seemed to be associated with fronto-limbic regions, especially for the ACC and hippocampus. However, there are still many efforts needed to clarify the highly promising biomarkers of MRI and MRS study in MDD, especially using the longitudinal study with randomized controlled design to investigate the specific biomarker regions for different kinds of therapeutic interventions, such as the pharmacological treatment, psychotherapeutic intervention and brain stimulation therapy.

\section{MDD biomarkers revealed by MEG}

The MEG can measure the brain activities using the algorithm to calculate the magnetic field caused by the electricity of brain activities to obtain the neuroimaging. The amount of MEG studies in MDD biomarker searching is relatively fewer than that of MRI studies. The electroconvulsive therapy might activate the parieto-temporal regions in the MEG of responding MDD patients, which suggested that the parietal lobe would influence the clinical response via the information processing and attention modulation. ${ }^{73}$ Another MEG study in drug-free MDD patients with high suicide risk showed the impaired phase-amplitude coupling in the limbic regions, such as the caudate and thalamus. The impaired coupling might influence the DLPFC to cause the alterations in the executive function and working memory. ${ }^{74}$ The higher scores of 
Lempel-Ziv complexity on MEG have been reported in MDD patients when compared to controls. ${ }^{75}$ A multimodal imaging of MDD patients, including MEG, showed that the subgenual ACC and amygdala might be the biomarker of MDD pathophysiology and ketamine treatment in MDD ${ }^{76}$ Another study showed that alpha connectivity between subgenual ACC and DLPFC would predict the better response of antidepressant treatment. ${ }^{77}$ The resting-state MEG study showed that hyperinterwines and hyper-integrates within the DLPFC and ACC might represent the MEG endophenotype of MDD. ${ }^{78}$ The ketamine treatment in MDD also would modulate the subgenual ACC-related connectivity network, including the limbic regions. ${ }^{79}$ The MEG-individual component analysis study also supported that subgenual ACC would be the core of biomarker network, which included the hippocampus and amygdala. ${ }^{80}$ The MEG dynamic causal modeling study demonstrated that DLPFC might be incapable to inhibit the amygdala and the subsequent enhanced amygdala-ACC and ACCDLPFC bottom-up signals would be associated with MDD symptoms. ${ }^{81}$ In spite of possible inconsistences, the major architecture of fronto-limbic biomarker network may be replicated in MEG study, especially the crucial role of ACC has been proved again.

\section{MDD biomarkers revealed by NIRS}

The NIRS use the near-infrared region of spectrum to measure the blood oxygenation, blood flow and brain activities. The MDD with suicidal ideation showed smaller hemodynamic changes in the DLPFC and OFC during verbal fluency test in the NIRS study. In addition, the hemodynamic changes in the DLPFC and OFC were negatively correlated with the severity of suicidal ideation. The DLPFC and OFC might represent the neural substrate for suicidal ideation. ${ }^{82}$ The blood flow in the frontal lobe would also be negatively associated the depression severity in another NIRS study ${ }^{83}$ Another study of NIRS in the dexamethasone suppression test of MDD patients showed that value of the center of gravity at the frontal lobe would be significantly greater in the non-suppressor group, which suggested that frontal lobe NIRS values might be representative of MDD patients with cortisol suppression impairments. ${ }^{84}$ The emotional face recognition task in MDD also revealed the importance of left prefrontal cortex due to the alterations of median value and ratios of oxygenated hemoglobin/deoxygenated hemoglobin, which suggested the weaker hemodynamic oscillations in the prefrontal cortex of MDD patients. ${ }^{85}$ The emotional Stroop task in MDD also found that an evoked wave in the left upper frontal cortex might be inversely correlated with the severity of depression. ${ }^{86}$ The antidepressant treatment seemed to influence the left inferior frontal cortex and temporal gyri in MDD patients, which suggested that prefrontal hemodynamic alterations might be the predictor of clinical responses to antidepressant treatment. ${ }^{87}$ A 1.5-years follow up study also confirmed the important role of frontal lobe in the state-dependent and trait biomarkers, which might be the inferior frontal cortex and middle frontal cortex respectively. ${ }^{88}$ Several studies of NIRS also supported the crucial role of frontal lobe or prefrontal cortex in the pathophysiology of MDD and might represent the biomarker for depression. ${ }^{89-92}$ It seemed that most NIRS studies in MDD emphasized the role of potentially promising biomarker in the frontal regions. The fronto-limbic network in the NIRS field of MDD probably would be more focused on the frontal component within this network.

\section{MDD biomarkers revealed by EEG}

The EEG is a traditional tool to understand the electric activities accompanied with brain activities. The cortical inhibition EEG signals, such as the N100 and LICI, in the DLPFC and other frontal regions might predict the treatment response to the magnetic seizure therapy. It suggested that stronger baseline inhibitory neurotransmission may represent the indicators. ${ }^{59}$ The EEG can assess the arousal level due to dysregulation of inner tension and might assist to decide which kind of patients might be responding to psychostimulant treatment. ${ }^{93}$ In addition, the vigilance regulation pattern of EEG has been applied to identify the MDD patients. ${ }^{94}$ The MDD responders of repetitive transcranial magnetic stimulation showed higher baseline and post-treatment working memory-related fronto-midline theta power and connectivity than non-responders. ${ }^{95}$ The alpha, theta and gamma oscillation waves of EEG can provide information on depressive states and recovery. The gamma rhythms can distinguish MDD patients from controls and bipolar disorder patients. In addition, the gamma rhythms can predict the treatment response of pharmacological and non-pharmacological treatment. ${ }^{96}$ Reduced gamma rhythms in the rostral ACC has been suggested as the biomarker for MDD. ${ }^{97}$ The resting-state EEG gamma power and theta-gamma coupling in frontal region might predict the treatment response of repetitive transcranial magnetic resonance treatment and the accompanied increases of gamma power in the frontal region would occur after treatment. ${ }^{98}$ The antidepressant responders of MDD had greater theta current density in the rostral ACC and OFC than non-responders. ${ }^{99}$ The EEG alpha asymmetry with greater right-lateralized activities has also been proposed as a kind of biomarker to identify MDD. ${ }^{100}$ The metaanalysis of EEG sleep pattern in MDD showed that increased rapid eye movement density and shortening slow wave sleep might represent the EEG sleep biomarkers of MDD. ${ }^{101}$ The REM sleep prefrontal theta cordance of MDD patients was 
also positively associated with the improvements in depression symptoms. In addition, the greater prefrontal theta cordance of REM sleep EEG might represent the biomarker for antidepressant treatment in MDD patients. ${ }^{102}$ Depressed adolescents also exhibited significantly increased slow wave activities of frontal regions during sleep, which were positively correlated with morbid thoughts. It represented the cortical regions of intense use or restructuring might be influenced in MDD. ${ }^{103}$ The prefrontal theta cordance, alpha and delta hemispheric asymmetries might also predict the antidepressant treatment in MDD. ${ }^{104}$ The theta cordance in the DLPFC and central brain regions also could predict the treatment response of brain stimulation..$^{105}$

In summary, due to the technique limitations of EEG, the findings would be localized on the cortex surface. The prefrontal cortex, such as ACC, DLPFC and OFC, might be crucial for the biomarker detection of MDD. The EEG biomarker characteristics of the prefrontal cortex included the theta density, theta cordance, alpha rhythm asymmetry, delta rhythm asymmetry, gamma rhythm and sleep EEG. The frontal part of fronto-limbic network has been proved in the EEG findings. However, the diversity of EEG parameters might be needed to be unified and processed to develop an applicable EEG model to identify MDD patients and the biomarker of treatment response in MDD.

\section{MDD biomarkers revealed by PET and SPECT}

The PET and SPECT can detect the neurotransmitter level using the radioligand labeling the brain region with targeted neurotransmitter receptors. The neuroticism personality, which were highly associated with MDD, was positively correlated with fronto-limbic serotonin 5HT-2A receptor binding potential. The fronto-limbic serotoninergic dysregulation might be vulnerable to MDD. ${ }^{106}$ The glucose metabolism study showed different modulation of brain glucose metabolisms by cognitive behavioral therapy and antidepressant. ${ }^{107}$ A serotonin PET study showed that the serotonin transporter binding potential was significantly lower in the forebrain, brainstem and putamen of MDD patients with suicide attempts. It also supported the serotoninergic dysregulation in MDD patients and suicide. ${ }^{108}$ Male MDD patients had significantly higher serotonin 5HT-1A binding potential over the raphe nuclei in the brainstem, DLPFC, ACC, other frontal regions, amygdala and hippocampus. ${ }^{109}$ The cerebral amyloid PET study showed that cerebral amyloidosis would occur in the hippocampus of MDD patients with mild cognitive impairment. ${ }^{110}$ The ketamine therapy in MDD would be associated with subgenual ACC glucose metabolism changes, which might suggest the crucial role of ACC for the glutamate modulation in the treatment of $\mathrm{MDD}{ }^{79}$ The decreased binding of serotonin transporter in the amygdala has also proposed as the potential biomarker for remission after antidepressant treatment. ${ }^{111}$ The binding potential of the neuroinflammation protein in subgenual prefrontal cortex and ACC might be increased in the unmedicated MDD patients, which also supported the crucial role of ACC from the viewpoint of neuro-inflmmation hypothesis in MDD. ${ }^{112}$ The subcallosal ACC glucose metabolism was significantly higher in non-responders than remitters after cognitive behavioral therapy and antidepressant treatment. ${ }^{113}$ The light therapy can decrease the serotonin transporter binding potential in the ACC of seasonal MDD during the winter season, which also supported the importance of ACC in the seasonal impacts of MDD via the serotoninergic regulation. ${ }^{114}$ The density of monoamine oxidase $\mathrm{A}$, a kind of enzyme to metabolize the serotonin, was found to be greater in the prefrontal cortex and ACC of patients with postpartum MDD. ${ }^{115}$ It emphasized the crucial role of ACC and frontal regions in the pathophysiology of MDD, including the seasonal and postpartum subtypes. The deep brain stimulation also reached remission in the treatment-resistant MDD patients with higher baseline prefrontal regional cerebral blood flow, which also suggested the predictive biomarker of prefrontal cortex for deep brain stimulation. ${ }^{116}$

The SPECT guided transcranial magnetic stimulation in the left DLPFC also improved the MDD symptoms, which also supported the DLPFC as the treatment biomarker for MDD. ${ }^{117}$ The lower serotonin transporter binding potential in the midbrain using SPECT modality would also be associated with heritability of MDD. ${ }^{118}$ The attenuation of negative attention bias in MDD after antidepressant treatment might be associated with serotonin transporter occupancy in the amygdala of a SPECT study in MDD, which suggested that antidepressant can relieve limbic elevated activities. ${ }^{119}$ The distribution volume ratio of serotonin transporter would be lower in the basal ganglia and midbrain. ${ }^{120}$ Another study of cerebral blood flow using SPECT also observed the decreased blood flow in the prefrontal cortex, especially the ACC, DLPFC and OFC, which also supported the critical role of prefrontal cortex in the blood flow biomarker of MDD. ${ }^{121}$ Other studies of SPECT in MDD seemed to focus on the midbrain and found the alterations in serotonin transporter occupancy of midbrain. ${ }^{122-124}$

In summary, the PET studies seemed to emphasize the ACC and other prefrontal cortex, such as the DLPFC and OFC in the biomarker search of MDD, especially for validation of the neuroinflammation theory, deep brain stimulation, antidepressant treatment effects, psychotherapy effects, seasonal effects and postpartum effects. The findings of limbic regions, such as amygdala and hippocampus, combined with the find- 
ings in the brainstem and midbrain, could be validated for the biomarkers of cognition impairment, antidepressant treatment and psychotherapy effects. For the SPECT study, most findings seemed to be focused on the midbrain, especially for the serotoninergic biomarker search in MDD. The frontal regions of fronto-limbic network might play a role in the biomarker of decreased cerebral blood flows. However, most studies of PET and SPECT were based on the region-of-interest method, which might be associated with selection bias and would influence the interpretations of the study results.

\section{CONCLUSION}

From the above literature review, the fronto-limbic network seemed the promising neuroimaging biomarkers in MDD, especially for MRI, MRS, MEG and PET study (Figure 1). The most promising region may be the ACC. The importance of frontal regions has also been confirmed in NIRS and EEG study. The frontal areas might inhibit the emotional responses from the limbic regions, such as amygdala and hippocampus. Further multimodal imaging study would be needed to elucidate the neuroimaging biomarker issue in MDD.

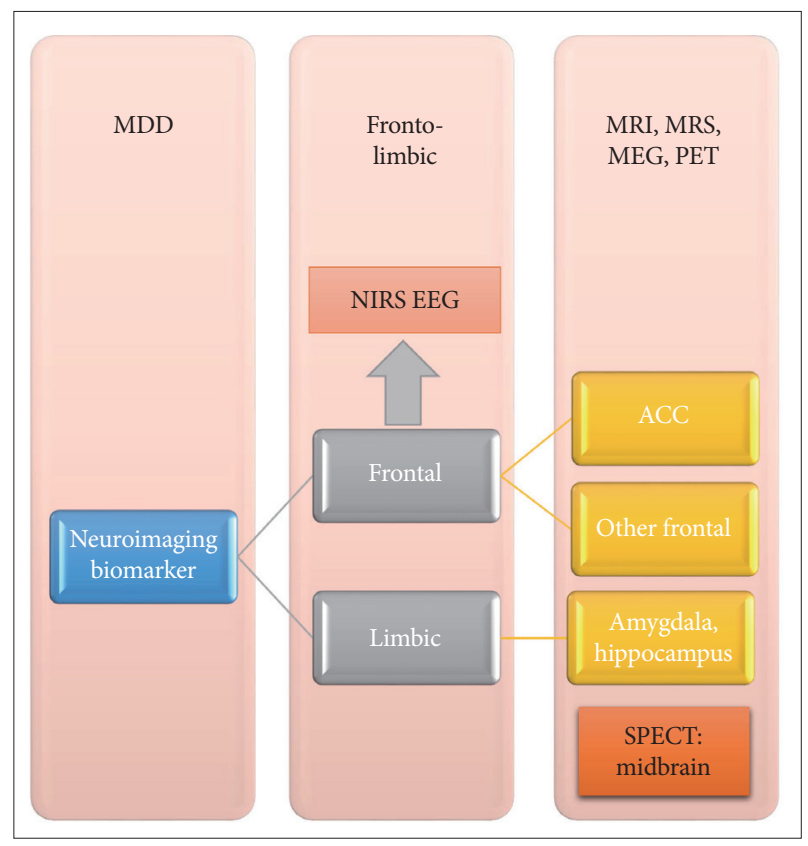

Figure 1. The fronto-limbic network and promising neuroimaging biomarker in MDD. The MRI, MRS, MEG, and PET study mentioned all the important parts involving the frontal and limbic regions. The most promising biomarker would be ACC. The NIRS and EEG also supported the crucial role of frontal regions. SPECT study revealed the clues of midbrain biomarker, which might be associated with the region-of-interest method. ACC: anterior cingulate cortex, EEG: electroencephalography, MEG: magnetoencephalography, MRI: magnetic resonance imaging, NIRS: near-infrared spectroscopy, PET: positron emission tomography, SPECT: singlephoton emission computed tomography.

\section{Conflicts of Interest}

The author has no potential conflicts of interest to disclose.

\section{ORCID iD}

Chien-Han Lai https://orcid.org/0000-0001-8259-1171

\section{REFERENCES}

1. Lai CH. Major depressive disorder: gender differences in symptoms, life quality, and sexual function. J Clin Psychopharmacol 2011;31:39-44.

2. Testa MA, Simonson DC. Assesment of quality-of-life outcomes. N Engl J Med 1996;334:835-840.

3. Naranjo CA, Tremblay LK, Busto UE. The role of the brain reward system in depression. Prog Neuropsychopharmacol Biol Psychiatry 2001;25:781-823.

4. Alexopoulos GS, Hoptman MJ, Kanellopoulos D, Murphy CF, Lim KO, Gunning FM. Functional connectivity in the cognitive control network and the default mode network in late-life depression. J Affect Disord 2012;139:56-65.

5. van Tol MJ, van der Wee NJ, van den Heuvel OA, Nielen MM, Demenescu LR, Aleman A, et al. Regional brain volume in depression and anxiety disorders. Arch Gen Psychiatry 2010;67:1002-1011.

6. Lai CH, Hsu YY, Wu YT. First episode drug-naive major depressive disorder with panic disorder: gray matter deficits in limbic and default network structures. Eur Neuropsychopharmacol 2010;20:676-682.

7. de Kwaasteniet B, Ruhe E, Caan M, Rive M, Olabarriaga S, Groefsema M, et al. Relation between structural and functional connectivity in major depressive disorder. Biol Psychiatry 2013;74:40-47.

8. Sheline YI. 3D MRI studies of neuroanatomic changes in unipolar major depression: the role of stress and medical comorbidity. Biol Psychiatry 2000;48:791-800.

9. Sheline YI, Barch DM, Price JL, Rundle MM, Vaishnavi SN, Snyder AZ, et al. The default mode network and self-referential processes in depression. Proc Natl Acad Sci U S A 2009;106:1942-1947.

10. Allman JM, Hakeem A, Erwin JM, Nimchinsky E, Hof P. The anterior cingulate cortex. The evolution of an interface between emotion and cognition. Ann N Y Acad Sci 2001;935:107-117.

11. Bush G, Luu P, Posner MI. Cognitive and emotional influences in anterior cingulate cortex. Trends Cogn Sci 2000;4:215-222.

12. Rushworth MF, Behrens TE, Rudebeck PH, Walton ME. Contrasting roles for cingulate and orbitofrontal cortex in decisions and social behaviour. Trends Cogn Sci 2007;11:168-176.

13. Yucel M, Wood SJ, Fornito A, Riffkin J, Velakoulis D, Pantelis C. Anterior cingulate dysfunction: implications for psychiatric disorders? J Psychiatry Neurosci 2003;28:350-354.

14. Devinsky O, Morrell MJ, Vogt BA. Contributions of anterior cingulate cortex to behaviour. Brain 1995;118(Pt 1):279-306.

15. Mayberg HS. Limbic-cortical dysregulation: a proposed model of depression. J Neuropsychiatry Clin Neurosci 1997;9:471-481.

16. Ressler KJ, Mayberg HS. Targeting abnormal neural circuits in mood and anxiety disorders: from the laboratory to the clinic. Nat Neurosci 2007;10:1116-1124.

17. Bae JN, MacFall JR, Krishnan KR, Payne ME, Steffens DC, Taylor WD. Dorsolateral prefrontal cortex and anterior cingulate cortex white matter alterations in late-life depression. Biol Psychiatry 2006; 60:1356-1363.

18. Frodl TS, Koutsouleris N, Bottlender R, Born C, Jager M, Scupin I, et al. Depression-related variation in brain morphology over 3 years: effects of stress? Arch Gen Psychiatry 2008;65:1156-1165.

19. Li CT, Lin CP, Chou KH, Chen IY, Hsieh JC, Wu CL, et al. Structural and cognitive deficits in remitting and non-remitting recurrent depression: a voxel-based morphometric study. Neuroimage 2010;50:347-356.

20. Liao C, Feng Z, Zhou D, Dai Q, Xie B, Ji B, et al. Dysfunction of frontolimbic brain circuitry in depression. Neuroscience 2012;201:231-238. 
21. Egger K, Schocke M, Weiss E, Auffinger S, Esterhammer R, Goebel G, et al. Pattern of brain atrophy in elderly patients with depression revealed by voxel-based morphometry. Psychiatry Res 2008;164:237-244.

22. Gatt JM, Nemeroff CB, Dobson-Stone C, Paul RH, Bryant RA, Schofield PR, et al. Interactions between BDNF Val66Met polymorphism and early life stress predict brain and arousal pathways to syndromal depression and anxiety. Mol Psychiatry 2009;14:681-695.

23. van Eijndhoven P, van Wingen G, Fernandez G, Rijpkema M, Verkes RJ, Buitelaar J, et al. Amygdala responsivity related to memory of emotionally neutral stimuli constitutes a trait factor for depression. Neuroimage 2011;54:1677-1684.

24. van Tol MJ, Demenescu LR, van der Wee NJ, Kortekaas R, Marjan MAN, Boer JA, et al. Functional magnetic resonance imaging correlates of emotional word encoding and recognition in depression and anxiety disorders. Biol Psychiatry 2012;71:593-602.

25. Herringa RJ, Birn RM, Ruttle PL, Burghy CA, Stodola DE, Davidson RJ, et al. Childhood maltreatment is associated with altered fear circuitry and increased internalizing symptoms by late adolescence. Proc Natl Acad Sci U S A 2013;110:19119-19124.

26. Gabbay V, Liebes L, Katz Y, Liu S, Mendoza S, Babb JS, et al. The kynurenine pathway in adolescent depression: preliminary findings from a proton MR spectroscopy study. Prog Neuropsychopharmacol Biol Psychiatry 2010;34:37-44.

27. Hahn T, Marquand AF, Ehlis AC, Dresler T, Kittel-Schneider S, Jarczok TA, et al. Integrating neurobiological markers of depression. Arch Gen Psychiatry 2011;68:361-368.

28. Lai CH. Patterns of cortico-limbic activations during visual processing of sad faces in depression patients: a coordinate-based metaanalysis. J Neuropsychiatry Clin Neurosci 2014;26:34-43.

29. Barch DM, Gaffrey MS, Botteron KN, Belden AC, Luby JL. Functional brain activation to emotionally valenced faces in school-aged children with a history of preschool-onset major depression. Biol Psychiatry 2012;72:1035-1042.

30. Gaffrey MS, Barch DM, Singer J, Shenoy R, Luby JL. Disrupted amygdala reactivity in depressed 4- to 6-year-old children. J Am Acad Child Adolesc Psychiatry 2013;52:737-746.

31. Stein JL, Medland SE, Vasquez AA, Hibar DP, Senstad RE, Winkler $\mathrm{AM}$, et al. Identification of common variants associated with human hippocampal and intracranial volumes. Nat Genet 2012;44:552-561.

32. Lan MJ, Rizk MM, Pantazatos SP, Rubin-Falcone H, Miller JM, Sublette ME, et al. Resting-state amplitude of low-frequency fluctuation is associated with suicidal ideation. Depress Anxiety 2019;36:433-441.

33. Arnone D, McKie S, Elliott R, Juhasz G, Thomas EJ, Downey D, et al. State-dependent changes in hippocampal grey matter in depression. Mol Psychiatry 2013;18:1265-1272.

34. Samann PG, Hohn D, Chechko N, Kloiber S, Lucae S, Ising M, et al. Prediction of antidepressant treatment response from gray matter volume across diagnostic categories. Eur Neuropsychopharmacol 2013;23:1503-1515.

35. Mannie ZN, Filippini N, Williams C, Near J, Mackay CE, Cowen PJ. Structural and functional imaging of the hippocampus in young people at familial risk of depression. Psychol Med 2014;44:2939-2948.

36. Maller JJ, Broadhouse K, Rush AJ, Gordon E, Koslow S, Grieve SM. Increased hippocampal tail volume predicts depression status and remission to anti-depressant medications in major depression. Mol Psychiatry 2018;23:1737-1744.

37. Shi XF, Forrest LN, Kuykendall MD, Prescot AP, Sung YH, Huber RS, et al. Anterior cingulate cortex choline levels in female adolescents with unipolar versus bipolar depression: a potential new tool for diagnosis. J Affect Disord 2014;167:25-29.

38. Riley CA, Renshaw PF. Brain choline in major depression: A review of the literature. Psychiatry Res Neuroimaging 2018; 271: 142-153.

39. Lai $\mathrm{CH}$. The regional homogeneity of cingulate-precuneus regions: The putative biomarker for depression and anxiety. J Affect Disord 2018;229:171-176.
40. Lai CH. Gray matter volume in major depressive disorder: a metaanalysis of voxel-based morphometry studies. Psychiatry Res 2013; 211:37-46.

41. Sundermann B, Olde Lutke Beverborg M, Pfleiderer B. Toward literature-based feature selection for diagnostic classification: a meta-analysis of resting-state fMRI in depression. Front Hum Neurosci 2014;8:692.

42. Boehringer A, Tost H, Haddad L, Lederbogen F, Wust S, Schwarz E, et al. Neural Correlates of the Cortisol Awakening Response in Humans. Neuropsychopharmacology 2015;40:2278-2285.

43. Sato JR, Moll J, Green S, Deakin JF, Thomaz CE, Zahn R. Machine learning algorithm accurately detects fMRI signature of vulnerability to major depression. Psychiatry Res 2015;233:289-291.

44. Lai CH, Wu YT. The alterations in regional homogeneity of parieto-cingulate and temporo-cerebellum regions of first-episode medicationnaive depression patients. Brain Imaging Behav 2016;10:187-194.

45. Argyelan M, Lencz T, Kaliora S, Sarpal DK, Weissman N, Kingsley $\mathrm{PB}$, et al. Subgenual cingulate cortical activity predicts the efficacy of electroconvulsive therapy. Transl Psychiatry 2016;6:e789.

46. Pirnia T, Joshi SH, Leaver AM, Vasavada M, Njau S, Woods RP, et al. Electroconvulsive therapy and structural neuroplasticity in neocortical, limbic and paralimbic cortex. Transl Psychiatry 2016;6:e832.

47. Serra-Blasco M, de Diego-Adelino J, Vives-Gilabert Y, Trujols J, Puigdemont D, Carceller-Sindreu M, et al. Naturalistic course of major depressive disorder predicted by clinical and structural neuroimaging data: a 5-year follow-up. Depress Anxiety 2016;33:1055-1064.

48. Boes AD, Uitermarkt BD, Albazron FM, Lan MJ, Liston C, PascualLeone A, et al. Rostral anterior cingulate cortex is a structural correlate of repetitive TMS treatment response in depression. Brain Stimul 2018;11:575-581.

49. Grieve SM, Korgaonkar MS, Gordon E, Williams LM, Rush AJ. Prediction of nonremission to antidepressant therapy using diffusion tensor imaging. J Clin Psychiatry 2016;77:e436-443.

50. Rzepa E, Fisk J, McCabe C. Blunted neural response to anticipation, effort and consummation of reward and aversion in adolescents with depression symptomatology. J Psychopharmacol 2017;31:303-311.

51. Brakowski J, Spinelli S, Dorig N, Bosch OG, Manoliu A, Holtforth MG, et al. Resting state brain network function in major depression Depression symptomatology, antidepressant treatment effects, future research. J Psychiatr Res 2017;92:147-159.

52. Bijsterbosch JD, Ansari TL, Smith S, Gauld O, Zika O, Boessenkool S, et al. Stratification of MDD and GAD patients by resting state brain connectivity predicts cognitive bias. Neuroimage Clin 2018;19:425-433.

53. Caceda R, Bush K, James GA, Stowe ZN, Kilts CD. Modes of resting functional brain organization differentiate suicidal thoughts and actions: a preliminary study. J Clin Psychiatry 2018;79. pii: 17m11901.

54. Gao S, Calhoun VD, Sui J. Machine learning in major depression: From classification to treatment outcome prediction. CNS Neurosci Ther 2018;24:1037-1052.

55. Zhang FF, Peng W, Sweeney JA, Jia ZY, Gong QY. Brain structure alterations in depression: Psychoradiological evidence. CNS Neurosci Ther 2018;24:994-1003.

56. Lai $\mathrm{CH}, \mathrm{Wu} \mathrm{YT}$. Alterations in white matter micro-integrity of the superior longitudinal fasciculus and anterior thalamic radiation of young adult patients with depression. Psychol Med 2014;44:2825-2832.

57. Lai $\mathrm{CH}, \mathrm{Wu} \mathrm{YT}$. The white matter microintegrity alterations of neocortical and limbic association fibers in major depressive disorder and panic disorder: the comparison. Medicine (Baltimore) 2016;95: e2982.

58. Lai CH. The neural markers of MRI to differentiate depression and panic disorder. Prog Neuropsychopharmacol Biol Psychiatry 2019; 91:72-78.

59. Sun Y, Farzan F, Mulsant BH, Rajji TK, Fitzgerald PB, Barr MS, et al. Indicators for remission of suicidal ideation following magnetic seizure therapy in patients with treatment-resistant depression. JAMA Psychiatry 2016;73:337-345. 
60. Korgaonkar MS, Grieve SM, Etkin A, Koslow SH, Williams LM. Using standardized fMRI protocols to identify patterns of prefrontal circuit dysregulation that are common and specific to cognitive and emotional tasks in major depressive disorder: first wave results from the iSPOT-D study. Neuropsychopharmacology 2013;38:863-871.

61. Vlasova RM, Siddarth P, Krause B, Leaver AM, Laird KT, St Cyr N, et al. Resilience and white matter integrity in geriatric depression. Am J Geriatr Psychiatry 2018;26:874-883.

62. Li B, Liu L, Friston KJ, Shen H, Wang L, Zeng LL, et al. A treatmentresistant default mode subnetwork in major depression. Biol Psychiatry 2013;74:48-54.

63. Wall CA, Croarkin PE, Maroney-Smith MJ, Haugen LM, Baruth JM, Frye MA, et al. Magnetic resonance imaging-guided, open-label, high-frequency repetitive transcranial magnetic stimulation for adolescents with major depressive disorder. J Child Adolesc Psychopharmacol 2016;26:582-589.

64. Oliveira-Maia AJ, Press D, Pascual-Leone A. Modulation of motor cortex excitability predicts antidepressant response to prefrontal cortex repetitive transcranial magnetic stimulation. Brain Stimul 2017; 10:787-794.

65. Stange JP, Bessette KL, Jenkins LM, Peters AT, Feldhaus C, Crane $\mathrm{NA}$, et al. Attenuated intrinsic connectivity within cognitive control network among individuals with remitted depression: Temporal stability and association with negative cognitive styles. Hum Brain Mapp 2017;38:2939-2954.

66. Vink JJT, Mandija S, Petrov PI, van den Berg CAT, Sommer IEC, Neggers SFW. A novel concurrent TMS-fMRI method to reveal propagation patterns of prefrontal magnetic brain stimulation. Hum Brain Mapp 2018;39:4580-4592.

67. Abdallah CG, Averill CL, Salas R, Averill LA, Baldwin PR, Krystal $\mathrm{JH}$, et al. Prefrontal connectivity and glutamate transmission: relevance to depression pathophysiology and ketamine treatment. Biol Psychiatry Cogn Neurosci Neuroimaging 2017;2:566-574.

68. Ribeiz SR, Duran F, Oliveira MC, Bezerra D, Castro CC, Steffens DC, et al. Structural brain changes as biomarkers and outcome predictors in patients with late-life depression: a cross-sectional and prospective study. PLoS One 2013;8:e80049.

69. Dai Q, Yin X, Li H, Feng Z. Orbito-frontal cortex mechanism of inhibition of return in current and remitted depression. Hum Brain Mapp 2018;39:2941-2954.

70. Bajs Janovic M, Kalember P, Janovic S, Hrabac P, Folnegovic Grosic P, Grosic V, et al. No change in $\mathrm{N}$-acetyl aspartate in first episode of moderate depression after antidepressant treatment: (1)H magnetic spectroscopy study of left amygdala and left dorsolateral prefrontal cortex. Neuropsychiatr Dis Treat 2014;10:1753-1762.

71. Brunoni AR, Baeken C, Machado-Vieira R, Gattaz WF, Vanderhasselt MA. BDNF blood levels after non-invasive brain stimulation interventions in major depressive disorder: a systematic review and meta-analysis. World J Biol Psychiatry 2015;16:114-122.

72. Oltedal L, Narr KL, Abbott C, Anand A, Argyelan M, Bartsch H, et al. Volume of the Human Hippocampus and Clinical Response Following Electroconvulsive Therapy. Biol Psychiatry 2018;84:574-581.

73. Zwanzger P, Klahn AL, Arolt V, Ruland T, Zavorotnyy M, Salzer J, et al. Impact of electroconvulsive therapy on magnetoencephalographic correlates of dysfunctional emotional processing in major depression. Eur Neuropsychopharmacol 2016;26:684-692.

74. Chattun MR, Zhang S, Chen Y, Wang Q, Amdanee N, Tian S, et al. Caudothalamic dysfunction in drug-free suicidally depressed patients: an MEG study. Eur Arch Psychiatry Clin Neurosci 2018 [Epub ahead of print].

75. Fernandez A, Al-Timemy AH, Ferre F, Rubio G, Escudero J. Complexity analysis of spontaneous brain activity in mood disorders: A magnetoencephalography study of bipolar disorder and major depression. Compr Psychiatry 2018;84:112-117.

76. Nugent AC, Farmer C, Evans JW, Snider SL, Banerjee D, Zarate CA Jr.
Multimodal imaging reveals a complex pattern of dysfunction in corticolimbic pathways in major depressive disorder. Hum Brain Mapp 2019;40:3940-3950.

77. Wang Q, Tian S, Tang H, Liu X, Yan R, Hua L, et al. Identification of major depressive disorder and prediction of treatment response using functional connectivity between the prefrontal cortices and subgenual anterior cingulate: A real-world study. J Affect Disord 2019; 252:365-372.

78. Tian S, Chattun MR, Zhang S, Bi K, Tang H, Yan R, et al. Dynamic community structure in major depressive disorder: A resting-state MEG study. Prog Neuropsychopharmacol Biol Psychiatry 2019;92:39-47.

79. Nugent AC, Robinson SE, Coppola R Zarate CA, Jr. Preliminary differences in resting state MEG functional connectivity pre- and postketamine in major depressive disorder. Psychiatry Res Neuroimaging 2016;254:56-66.

80. Nugent AC, Robinson SE, Coppola R, Furey ML, Zarate CA Jr. Group differences in MEG-ICA derived resting state networks: Application to major depressive disorder. Neuroimage 2015;118:1-12.

81. Lu Q, Li H, Luo G, Wang Y, Tang H, Han L, et al. Impaired prefrontal-amygdala effective connectivity is responsible for the dysfunction of emotion process in major depressive disorder: a dynamic causal modeling study on MEG. Neurosci Lett 2012;523:125-130.

82. Pu S, Nakagome K, Yamada T, Yokoyama K, Matsumura H, Yamada $\mathrm{S}$, et al. Suicidal ideation is associated with reduced prefrontal activation during a verbal fluency task in patients with major depressive disorder. J Affect Disord 2015;181:9-17.

83. Kawano M, Kanazawa T, Kikuyama H, Tsutsumi A, Kinoshita S, Kawabata Y, et al. Correlation between frontal lobe oxy-hemoglobin and severity of depression assessed using near-infrared spectroscopy. J Affect Disord 2016;205:154-158.

84. Kinoshita S, Kanazawa T, Kikuyama H, Yoneda H. Clinical application of DEX/CRH test and multi-channel NIRS in patients with depression. Behav Brain Funct 2016;12:25.

85. Gao L, Cai Y, Wang H, Wang G, Zhang Q, Yan X. Probing prefrontal cortex hemodynamic alterations during facial emotion recognition for major depression disorder through functional near-infrared spectroscopy. J Neural Eng 2019;16:026026.

86. Nishizawa Y, Kanazawa T, Kawabata Y, Matsubara T, Maruyama S, Kawano M, et al. fNIRS assessment during an emotional stroop task among patients with depression: replication and extension. Psychiatry Investig 2019;16:80-86.

87. Yamagata B, Yamanaka K, Takei Y, Hotta S, Hirano J, Tabuchi H, et al. Brain functional alterations observed 4-weekly in major depressive disorder following antidepressant treatment. J Affect Disord 2019;252:25-31.

88. Satomura Y, Sakakibara E, Takizawa R, Koike S, Nishimura Y, Sakurada $\mathrm{H}$, et al. Severity-dependent and -independent brain regions of major depressive disorder: A long-term longitudinal near-infrared spectroscopy study. J Affect Disord 2019;243:249-254.

89. Liu X, Sun G, Zhang X, Xu B, Shen C, Shi L, et al. Relationship between the prefrontal function and the severity of the emotional symptoms during a verbal fluency task in patients with major depressive disorder: a multi-channel NIRS study. Prog Neuropsychopharmacol Biol Psychiatry 2014;54:114-121.

90. Matsubara T, Matsuo K, Nakashima M, Nakano M, Harada K, Watanuki T, et al. Prefrontal activation in response to emotional words in patients with bipolar disorder and major depressive disorder. Neuroimage 2014;85 Pt 1:489-497.

91. Takei Y, Suda M, Aoyama Y, Sakurai N, Tagawa M, Motegi T, et al. Near-infrared spectroscopic study of frontopolar activation during face-to-face conversation in major depressive disorder and bipolar disorder. J Psychiatr Res 2014;57:74-83.

92. Akashi H, Tsujii N, Mikawa W, Adachi T, Kirime E, Shirakawa O. Prefrontal cortex activation is associated with a discrepancy between self- and observer-rated depression severities of major depressive 
disorder: a multichannel near-infrared spectroscopy study. J Affect Disord 2015;174:165-172.

93. Hegerl U, Hensch T. Why do stimulants not work in typical depression? Aust N Z J Psychiatry 2017;51:20-22.

94. Olbrich S, Sander C, Minkwitz J, Chittka T, Mergl R, Hegerl U, et al. EEG vigilance regulation patterns and their discriminative power to separate patients with major depression from healthy controls. Neuropsychobiology 2012;65:188-194.

95. Bailey NW, Hoy KE, Rogasch NC, Thomson RH, McQueen S, Elliot $\mathrm{D}$, et al. Responders to rTMS for depression show increased frontomidline theta and theta connectivity compared to non-responders. Brain Stimul 2018;11:190-203.

96. Fitzgerald PJ, Watson BO. Gamma oscillations as a biomarker for major depression: an emerging topic. Transl Psychiatry 2018;8:177.

97. Pizzagalli DA, Peccoralo LA, Davidson RJ, Cohen JD. Resting anterior cingulate activity and abnormal responses to errors in subjects with elevated depressive symptoms: a 128-channel EEG study. Hum Brain Mapp 2006;27:185-201.

98. Noda Y, Zomorrodi R, Saeki T, Rajji TK, Blumberger DM, Daskalakis ZJ, et al. Resting-state EEG gamma power and theta-gamma coupling enhancement following high-frequency left dorsolateral prefrontal rTMS in patients with depression. Clin Neurophysiol 2017; 128:424-432.

99. Korb AS, Hunter AM, Cook IA, Leuchter AF. Rostral anterior cingulate cortex theta current density and response to antidepressants and placebo in major depression. Clin Neurophysiol 2009;120:1313-1319.

100. Gordon E, Palmer DM, Cooper N. EEG alpha asymmetry in schizophrenia, depression, PTSD, panic disorder, ADHD and conduct disorder. Clin EEG Neurosci 2010;41:178-183.

101. Pillai V, Kalmbach DA, Ciesla JA. A meta-analysis of electroencephalographic sleep in depression: evidence for genetic biomarkers. Biol Psychiatry 2011;70:912-919.

102. Adamczyk M, Gazea M, Wollweber B, Holsboer F, Dresler M, Steiger A, et al. Cordance derived from REM sleep EEG as a biomarker for treatment response in depression--a naturalistic study after antidepressant medication. J Psychiatr Res 2015;63:97-104.

103. Tesler N, Gerstenberg M, Franscini M, Jenni OG, Walitza S, Huber R. Increased frontal sleep slow wave activity in adolescents with major depression. Neuroimage Clin 2016;10:250-256.

104. Baskaran A, Farzan F, Milev R, Brenner CA, Alturi S, Pat McAndrews $\mathrm{M}$, et al. The comparative effectiveness of electroencephalographic indices in predicting response to escitalopram therapy in depression: A pilot study. J Affect Disord 2018;227:542-549.

105. Hunter AM, Nghiem TX, Cook IA, Krantz DE, Minzenberg MJ, Leuchter AF. Change in quantitative EEG theta cordance as a potential predictor of repetitive transcranial magnetic stimulation clinical outcome in major depressive disorder. Clin EEG Neurosci 2018;49: 306-315.

106. Frokjaer VG, Mortensen EL, Nielsen FA, Haugbol S, Pinborg LH, Adams $\mathrm{KH}$, et al. Frontolimbic serotonin 2A receptor binding in healthy subjects is associated with personality risk factors for affective disorder. Biol Psychiatry 2008;63:569-576.

107. McGrath CL, Kelley ME, Holtzheimer PE, Dunlop BW, Craighead WE, Franco AR, et al. Toward a neuroimaging treatment selection biomarker for major depressive disorder. JAMA Psychiatry 2013;70:821-829.

108. Nye JA, Purselle D, Plisson C, Voll RJ, Stehouwer JS, Votaw JR, et al. Decreased brainstem and putamen SERT binding potential in depressed suicide attempters using [11C]-zient PET imaging. Depress Anxiety 2013;30:902-907.

109. Kaufman J, Sullivan GM, Yang J, Ogden RT, Miller JM, Oquendo MA, et al. Quantification of the serotonin 1A receptor using PET: identifi- cation of a potential biomarker of major depression in males. Neuropsychopharmacology 2015;40:1692-1699.

110. Wu KY, Lin KJ, Chen CH, Chen CS, Liu CY, Huang SY, et al. Diversity of neurodegenerative pathophysiology in nondemented patients with major depressive disorder: Evidence of cerebral amyloidosis and hippocampal atrophy. Brain Behav 2018;8:e01016.

111. Ananth MR, DeLorenzo C, Yang J, Mann JJ, Parsey RV. Decreased pretreatment amygdalae serotonin transporter binding in unipolar depression remitters: a prospective PET study. J Nucl Med 2018;59: 665-670.

112. Richards EM, Zanotti-Fregonara P, Fujita M, Newman L, Farmer C, Ballard ED, et al. PET radioligand binding to translocator protein (TSPO) is increased in unmedicated depressed subjects. EJNMMI Res 2018;8:57.

113. McGrath CL, Kelley ME, Dunlop BW, Holtzheimer PE 3rd, Craighead WE, Mayberg HS. Pretreatment brain states identify likely nonresponse to standard treatments for depression. Biol Psychiatry 2014; 76:527-535.

114. Harrison SJ, Tyrer AE, Levitan RD, Xu X, Houle S, Wilson AA, et al. Light therapy and serotonin transporter binding in the anterior cingulate and prefrontal cortex. Acta Psychiatr Scand 2015;132:379-388.

115. Sacher J, Rekkas PV, Wilson AA, Houle S, Romano L, Hamidi J, et al. Relationship of monoamine oxidase-A distribution volume to postpartum depression and postpartum crying. Neuropsychopharmacology 2015;40:429-435.

116. Conen S, Matthews JC, Patel NK, Anton-Rodriguez J, Talbot PS. Acute and chronic changes in brain activity with deep brain stimulation for refractory depression. J Psychopharmacol 2018;32:430-440.

117. Jha S, Chadda RK, Kumar N, Bal CS. Brain SPECT guided repetitive transcranial magnetic stimulation (rTMS) in treatment resistant major depressive disorder. Asian J Psychiatr 2016;21:1-6.

118. Hsieh PC, Chen KC, Yeh TL, Lee IH, Chen PS, Yao WJ, et al. Lower availability of midbrain serotonin transporter between healthy subjects with and without a family history of major depressive disorder - a preliminary two-ligand SPECT study. Eur Psychiatry 2014;29:414-418.

119. Ruhe HG, Koster M, Booij J, van Herk M, Veltman DJ, Schene AH. Occupancy of serotonin transporters in the amygdala by paroxetine in association with attenuation of left amygdala activation by negative faces in major depressive disorder. Psychiatry Res 2014;221:155-161.

120. Newberg AB, Amsterdam JD, Wintering N, Shults J. Low brain serotonin transporter binding in major depressive disorder. Psychiatry Res 2012;202:161-167.

121. Nagafusa Y, Okamoto N, Sakamoto K, Yamashita F, Kawaguchi A, Higuchi T, et al. Assessment of cerebral blood flow findings using 99mTc-ECD single-photon emission computed tomography in patients diagnosed with major depressive disorder. J Affect Disord 2012;140:296-299.

122. Ruhe HG, Ooteman W, Booij J, Michel MC, Moeton M, Baas F, et al. Serotonin transporter gene promoter polymorphisms modify the association between paroxetine serotonin transporter occupancy and clinical response in major depressive disorder. Pharmacogenet $\mathrm{Ge}$ nomics 2009;19:67-76.

123. Ruhe HG, Booij J, v Weert HC, Reitsma JB, Franssen EJ, Michel MC, et al. Evidence why paroxetine dose escalation is not effective in major depressive disorder: a randomized controlled trial with assessment of serotonin transporter occupancy. Neuropsychopharmacology 2009;34:999-1010.

124. Ruhe HG, Booij J, Reitsma JB, Schene AH. Serotonin transporter binding with [123I]beta-CIT SPECT in major depressive disorder versus controls: effect of season and gender. Eur J Nucl Med Mol Imaging 2009;36:841-849. 